\title{
Genome-wide identification and analysis of the CNGC gene family in maize
}

\author{
Lidong Hao ${ }^{\text {Corresp.., } 1}$, Xiuli Qiao ${ }^{\text {Corresp. } 2}$ \\ ${ }^{1}$ College of Agriculture and Hydraulic Engineering, Suihua University, Suihua, HeiLongjiang province, China \\ 2 College of Food and Pharmaceutical Engineering, Suihua University, Suihua, HeiLongjiang province, China \\ Corresponding Authors: Lidong Hao, Xiuli Qiao \\ Email address: haolidong1987@163.com, shxynxhld@163.com
}

As one of the non-selective cation channel gene families, the cyclic nucleotide-gated channel (CNGC) gene family plays a vital role in plant physiological processes that are related to signal pathways, plant development, and environmental stresses.However, genome-wide identification and analysis of the CNGC gene family in maize has not yet been undertaken. In the present study, twelve $Z m C N G C$ genes were identified in the maize genome, which were unevenly distributed on chromosomes $1,2,4,5,6,7$, and 8 . They were classified into five major groups, which were Groups I, II, III, IVa, and IVb. Phylogenetic analysis showed that gramineous plant CNGC genes expanded unequally during evolution. Group IV CNGC genes emerged first, whereas Groups I and II appeared later. Prediction analysis of cis-acting regulatory elements showed that 137 putative ciselements were related to hormone-response, abiotic stress, and organ development. Furthermore, 120 protein pairs were predicted to interact with the $12 \mathrm{ZmCNGC}$ proteins and other maize proteins. The expression profiles of the ZmCNGC genes were expressed in tissue-specific patterns. These results provide important information that will increase our understanding of the CNGC gene family in maize and other plants. 
1 Genome-wide identification and analysis of the $C N G C$ gene gamily in maize

2 Lidong $\mathrm{Hao}^{1}$, Xiuli Qiao ${ }^{2 *}$

$3{ }^{1}$ College of Agriculture and Hydraulic Engineering, Suihua University, 18 Huanghe Road, Suihua,

4 Heilongjiang Province, 152000, PR China

$5{ }^{2}$ College of Food and Pharmaceutical Engineering, Suihua University, 18 Huanghe Road, Suihua,

6 Heilongjiang Province, 152000, PR China

7

$8 *$ Corresponding author:

9 Xiuli Qiao

10 Email: $\underline{\text { shxynxhld@,163.com }}$ 


\section{Abstract}

13 As one of the non-selective cation channel gene families, the cyclic nucleotide-gated channel 14 (CNGC) gene family plays a vital role in plant physiological processes that are related to signal 15 pathways, plant development, and environmental stresses. However, genome-wide identification and analysis of the $C N G C$ gene family in maize has not yet been undertaken. In the present study, twelve $Z m C N G C$ genes were identified in the maize genome, which were unevenly distributed on chromosomes $1,2,4,5,6,7$, and 8 . They were classified into five major groups, which were Groups I, II, III, IVa, and IVb. Phylogenetic analysis showed that gramineous plant $C N G C$ genes expanded unequally during evolution. Group IV $C N G C$ genes emerged first, whereas Groups I and II appeared later. Prediction analysis of cis-acting regulatory elements showed that 137 putative cis-elements were related to hormone-response, abiotic stress, and organ development. Furthermore, 120 protein pairs were predicted to interact with the $12 \mathrm{ZmCNGC}$ proteins and other maize proteins. The expression profiles of the $Z m C N G C$ genes were expressed in tissue-specific patterns. These results provide important information that will increase our understanding of the $C N G C$ gene family in maize and other plants. 


\section{INTRODUCTION}

Organism evolution has led to the formation of complex nutrient absorption and transport systems, including ion channels, ion pumps, and carriers. It has been previously shown that these systems respond to endogenous and abiotic stimuli (Saand et al. 2015b). The cyclic nucleotide-gated channel (CNGC) is a $\mathrm{Ca}^{2+}$-permeable cation transport channel, and it has been suggested that it is one of the fundamental mechanisms in organism systems (Yuen and Christopher 2013; Nawaz et al. 2014). Secondary messengers, such as cyclic nucleotide monophosphates (3',5'-cAMP and 3',5'-cGMP) and $\mathrm{Ca}^{2+} /$ calmodulin (CaM), can regulate CNGCs by acting as molecular switches. The CNGCs are activated by directly binding cyclic nucleotides and are inhibited when CaM binds to the CaM binding domain (Saand et al. 2015b; Borsics et al. 2007; Defalco et al. 2016; Kaplan et al. 2007).

In plants, CNGCs are composed of six transmembrane (TM) domains and one pore region between the fifth and sixth TM domains. The cyclic nucleotide-binding domain (CNBD) is a highly conserved region and has a phosphate-binding cassette (PBC) and a hinge region (Saand et al. 2015b). Unfortunately, although the existence of these domains is necessary for CNGC function, they cannot be used to identify a CNGC protein because other ion transporters such as potassium AKT/KAT channels (Shaker type) also contain both a CNBD and a TM domain (Su et al. 2001; Chérel 2004). However, previous studies have proposed that a plant CNGC-specific motif, [LIMV0]-X(2)-[GSANCR]-X-[FVIYASCL]-X-G-X(0,1)-X(0,1)-[EDAQGH]-L-[LIVFA]-X[WRCMLS0]-X-[LMSIQAFT0]-X(7,37)-[SAC]-X(9)-[VTIALMS]-X(0,1)-[EQDN]-[AGSVT][FYL]-X-[LIVF], in the PBC and hinge region within the CNBD of CNGC proteins only exists in plant CNGCs and does not occur in other ion transporters (Zelman et al. 2013; Saand et al. 2015b). The first plant CNGC was identified in Hordeum vulgare and named HvCBTl (Schuurink et al. 1998). The use of bioinformatics tools has led to the identification of the $C N G C$ gene family members in Arabidopsis (20), rice (16) and other plants (18 in tomato and 26 in Brassica oleracea) (Bridges et al. 2005; Nawaz et al. 2014; Ward et al. 2009; Zelman et al. 2012; Saand et al. 2015a; Chen et al. 2015; Zelman et al. 2013; Guo et al. 2017; Kakar et al. 2017).

Previous studies have shown that CNGCs are key components of plant development. Most $C N G C$ s 
57 have been characterized by genetic methods. They have been shown to play vital roles related to plant physiological and molecular functions, such as multiple physiological processes involved in signal pathways, plant development, and responses to environmental stresses. For example, Arabidopsis $C N G C 7$ and $C N G C 8$ are essential for male reproductive fertility (Tunc-Ozdemir et al. 2013a); $C N G C 16$ and $C N G C 18$ participate in pollen development (Tunc-Ozdemir et al. 2013b; Frietsch et al. 2007; Gao et al. 2016); AtCNGC2 is involved in jasmonic acid induced apoplastic $\mathrm{Ca}^{2+}$ influx in epidermal cells (Lu et al. 2015; Wang et al. 2017); and Arabidopsis CNGC6, CNGC19, and CNGC20 are involved in abiotic stress response (Kugler et al. 2009; Gao et al. 2012). Arabidopsis CNGC structures have six TM domains and a pore domain. They also possess a cyclic nucleotide-binding domain and CaM-binding domain in the C-terminus. These various domains have diverse functions (Talke et al. 2003; Chin et al. 2009a; Hua et al. 2003; Köhler and Neuhaus 2000). For example, AtCNGC2 plays a key role in stress signaling pathways, including changes to the cytosolic free $\mathrm{Ca}^{2+}$ in Arabidopsis. In contrast, $C N G C 4$ is permeable to $\mathrm{K}^{+}$and $\mathrm{Na}^{+}$, and is activated by both cGMP and cAMP (Balague 2003; Ali et al. 2007).

In recent years, efforts had been made to study the $C N G C$ gene family in plants. However, there have been few studies on the maize $C N G C$ gene family, even though maize is an important food crop and a source of industrial materials worldwide. This study used maize genome-wide sequence information, research information on Arabidopsis and rice CNGC families, and comprehensive bioinformatics analysis techniques to conduct a genome-wide identification of CNGCs in maize. To the best of our knowledge, this is the first systematic study of $C N G C$ genes in maize and provides the basis for future research on the $Z m C N G C$ gene family.

\section{MATERIALS AND METHODS}

\section{Identification of $C N G C$ genes in the maize genome}

A total of 20 Arabidopsis and 16 rice $C N G C$ protein sequences were retrieved from the Arabidopsis Information Resource (TAIR10) database (http://www.arabidopsis.org/) and the Rice Genome Annotation Project (RGAP) database (http://rice.plantbiology.msu.edu/), respectively. 
84

This information was then used to identify the $C N G C$ genes in maize. Two methods were utilized to search the maize protein sequences. One used a Hidden Markov Model (HMM) to search against maize protein sequences and the other used the local BLASTP method with a threshold e-value $<$ 1e-5. After the searches were conducted, a manual correction was performed to remove any redundancy and proteins without PBC and hinge regions within the CNBD of the CNGC proteins. To further confirm whether the ZmCNGC proteins contained the CNBD domain, the putative ZmCNGC protein sequences were submitted to the SMART (Letunic and Bork 2018) and NCBICDD databases (Marchler-Bauer et al. 2017). The proteins without CNBD domains or with amino acid (aa) numbers below 200 were removed and the ZmCNGCs confirmed. Another 11 gramineae plant CNGCs were identified by applying the same method as that described above.

The PI (theoretical isoelectric point), MW (molecular weight), and GRAVY (grand average of hydropathy) of the ZmCNGCs were predicted by ExPASy (Artimo et al. 2012). CELLO v.2.5 software was used to predict the subcellular location of the ZmCNGCs (Yu et al. 2010). Information about the chromosome distribution of ZmCNGCs and genetic sequences, such as DNA sequences, CDS, cDNA, and up-stream 1500 base pair (bp) DNA sequences obtained from a BLASTN search of the Ensembl Plant database (Bolser et al. 2016).

\section{Multiple alignments, phylogenetic analysis and gene duplication analysis}

Multiple sequence alignments were performed using the T-COFFEE method (Di Tommaso et al. 2011) and visualized by ESPript using the default program setting (Robert and Gouet 2014). A maximum likelihood (ML) phylogenetic tree was constructed using the MEGA 7 software program with 1,000 bootstrap replications and the Jones-Taylor-Thornton model (Kumar et al. 2016). To further validate the accuracy of the ML tree, an un-rooted phylogenetic tree was constructed with 1,000 bootstrap replications using the MEGA 7 software program and was based on full-length protein sequence alignments. The ML analysis of 12 gramineae plants was performed using the IQTree program with the $\mathrm{LG}+\mathrm{G} 4$ and state frequencies were determined from the amino acid matrix and other default parameters (Lam-Tung et al. 2015). The tree was 
111 visualized by Evolview online (He et al. 2016). Segmental duplication between maize genes and 112 the synteny block between maize and Sorghum, and rice and Brachypodium were obtained from 113 the Plant Genome Duplication database (Lee et al. 2013). The substitution rates (Ka/Ks) for 114 duplication events were calculated by the DnaSP v5 software program (Librado and Rozas 2009), 115 and the divergence times (Mya) were calculated using the formula: Mya $=\mathrm{Ks} / 2 \lambda \times 10^{-6}$, where $\lambda$ $116=6.5 \times 10^{-9}($ Lynch and Conery 2000$)$.

117

\section{Gene structure and conserved motif analyses}

119

120

121

122

123

124

125

126

127

128

129

130

131

132

\section{3}

134

135

136

137

The gene structures (exon-intron) of the $Z m C N G C$ genes were determined by the Gene Structure Display Server (Hu et al. 2015) using the CDS and DNA sequences from the $Z m C N G C$ genes. The conserved motifs of the ZmCNGC proteins were subjected to the MEME Suite web server (Bailey et al. 2015) with the maximum number of motifs set at 10 and an optimum width range for the motifs of $6-200$ aa.

Cis-acting regulatory elements and the prediction of protein-protein interaction in ZmCNGCs

The up-stream 1,500bp DNA sequences in the $Z m C N G C$ genes were used to locate cis-acting regulatory elements by the 'Signal Scan Search' programs in the NEW PLACE database (Higo et al. 1999). The interactions between ZmCNGCs and other maize proteins were predicted using the STRING 10 online program (Szklarczyk et al. 2017) and visualized by the Cytoscape v3.4.0 software program (Shannon et al. 2003).

\section{$Z m C N G C$ gene expression profiles and network interaction analysis}

To understand the expression of $Z m C N G C$ genes in different tissues, two high-throughput datasets for of maize were obtained from the Expression Atlas datasets (https://www.ebi.ac.uk/gxa/home/) under accession numbers E-MTAB-3826 and E-MTAB-439. These data were used to analyze the expression of $Z m C N G C$ genes in six different tissues (ear, embryo, endosperm, pollen, root, and 
138

139

140

141

142

143

144

145

146

147

148

149

150

151

152

153

154

155

156

157

158

159

160

161

162

163

164 tassel) and at different developmental stages (embryo, endosperm, and seed). The FPKM values were used to calculate the $Z m C N G C$ genes expressions and these were visualized by OmicShare tools, which is a free online platform for data analysis (http://www.omicshare.com/tools).

\section{RESULTS}

\section{Identification of the $C N G C$ genes in maize}

A total of 20 Arabidopsis and 16 rice CNGC protein sequences which had been BLAST aligned against maize protein sequences, were used to obtain an overview of the $C N G C$ genes in maize. After BLAST alignment, 18 putative $Z m C N G C$ genes were identified in the maize genome. The 18 putative $Z m C N G C$ genes were confirmed by using SMART and NCBI CDD to determine whether they contained the CNGC-specific domains (CNBD and TM). After removing redundant genes, $12 \mathrm{ZmCNGC}$ genes were finally identified, which was lower than the number of $C N G C$ genes in rice and Arabidopsis (Paterson et al. 2004; Yu et al. 2005). The predicted ZmCNGC genes were designated as $\mathrm{ZmCNGC1}$ to $\mathrm{ZmCNGC12}$ based on their family classification (Table 1). To further confirm the existence of the $\mathrm{ZmCNGCs,} \mathrm{we} \mathrm{identified} \mathrm{all} \mathrm{the} \mathrm{expressed} \mathrm{sequence} \mathrm{tags}$ (ESTs) that had aligned to the ZmCNGC genes by using the BLASTN program provided by the NCBI. The results demonstrated that only ZmCNGC3 had showed no EST hits, whereas the other ZmCNGCs had more than 13 representative matches to ESTs. Five of them were located on chromosome 5, whereas the others were unevenly located on chromosomes $1,2,4,6$, 7, and 8 . The characteristic features of these $12 \mathrm{ZmCNGC}$ genes are listed in Table 1. The ZmCNGC protein lengths ranged from 326 to 745 aa with an average of 612 aa. The molecular weights of these proteins ranged from $38.63 \mathrm{kDa}(\mathrm{ZmCNGC})$ to $85.52 \mathrm{kDa}(\mathrm{ZmCNGC})$ and the calculated $\mathrm{pI}$ values ranged from 8.92 (ZmCNGC4) to 9.75 (ZmCNGC12). Subcellular localization prediction analysis showed that all the ZmCNGCs were localized in the plasma membrane except for ZmCNGC3 which was localized in the nuclear fraction. These results are similar to Arabidopsis (Lemtiri-Chlieh and Berkowitz 2004). 
165

166

167

168

169

170

171

172

173

174

175

176

177

178

179

180

181

182

183

184

185

186

187

188

189

190

191

\section{Multiple alignments of maize CNGCs and potassium AKT/KAT channel genes}

Many ion transporters other than CNGCs also have a CNBD in the C-terminus and a hexa-TM in the N-terminus. For example, potassium AKT/KAT channels (Shaker type) also contain a CNBD and a TM domain. All AKT/ KAT-type channels consist of six TM regions with one P region (Su et al. 2001). Therefore, 11 maize AKT/KAT proteins were obtained from the NCBI resource and aligned with the $12 \mathrm{ZmCNGC}$ protein sequences (Supplemental File 1). The results showed that the proteins were highly conserved and that all of them contained six TM domains (S1-S6) and a pore region. The $\mathrm{PBC}$ and hinge domain were also highly conserved. A ML phylogenetic tree indicated that maize CNGC and AKT/KAT-type channel genes were clustered into two separate sections (Supplemental File1).

The CNBD is a gene structural feature element in plant CNGCs that contains the PBC and hinge region (Diller et al. 2001). Figure 1 illustrates that within the maize PBCs, a conserved phenylalanine $(\mathrm{F})$, a stabilizing glycine $(\mathrm{G})$ and an acidic residue (D or E), and two aliphatic leucines (L) were 100\% conserved inside the PBCs. Additionally, aromatic phenylalanine (F) and leucine $(\mathrm{L})$ were $100 \%$ conserved within the hinge region. These two conserved regions occurred between the CNBD and CaMBD regions. Based on corresponding alignments with other plants, a stringent motif (L-X(2)-G-[ED]-ELL-[TSG]-W-[ACY]-L-X(10,20)-[SA]-X-T-X(7)-[EQ]-[AG]F-X-L) which was recognized in the 12 maize CNGCs that included the PBC and hinge domain, was found to be consistent with other plant species (Saand et al. 2015b; Nawaz et al. 2014). The maize, rice, and Arabidopsis CNGCs were also aligned. The results showed that no positions were specific to the maize CNGC consensus, which suggested that the PBCs and hinge domain were highly conserved among plants (Supplemental File 2).

\section{Phylogenetic and duplication analyses of ZmCNGCs}

Large phylogenetic trees with minimal homologous characters increase the likelihood of confounding relationships between different species. Therefore, we constructed a ML phylogenetic tree based on the alignment of CNGC proteins from 12 gramineae plants. The tree 
192 included 24 CNGC proteins in Aegilops tauschii, 16 in Brachypodium distachyon, 20 in Hordeum 193 vulgare, 23 in Leersia perrieri, 21 in Nicotiana attenuata, 16 in Oryza sativa, 28 in Setaria italic, 19413 in Sorghum bicolor, 79 in Triticum aestivum, 21 in Triticum urartu, and 12 maize CNGCs 195 identified by this study (Supplemental File 5: Table 1). The phylogenetic tree (Supplemental File 196 4), was used to cluster these plant CNGC proteins into six groups, which were named Groups I, II, III, IV, IVa, and IVb, all of which had significant bootstrap values. The results showed that the CNGC proteins from B. distachyon, O. sativa, S. bicolor, and maize did not cluster in Group IV, and the $N$. attenuata CNGCs did not cluster in Group I. Additionally, the number in each group was unevenly distributed. Group IV was the largest with 86 genes, followed by 69 in Group III, 201 44 in Group II, 33 in Group IVb, 25 in Group I, and 16 in Group IVa. These data demonstrate that gramineae plant CNGC gene expansion appeared to have occurred unequally during evolution.

To better understand the evolutionary relationship among CNGC proteins, a ML phylogenetic tree was created based on the full-length protein alignments of $12 \mathrm{ZmCNGCs}, 19 \mathrm{AtCNGCs}$ and 16 OsCNGCs (Nawaz et al. 2014; Maser et al. 2001). The phylogenetic tree shows that the 47 CNGC proteins could be classified into five groups with significant bootstrap values (Figure 2). These were named Groups I, II, III, IVa, and IVb. This was consistent with what has been previously reported for flowering plant CNGCs (Saand et al. 2015b). Group I contained three maize CNGC

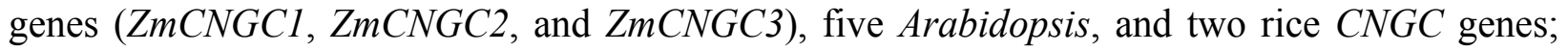

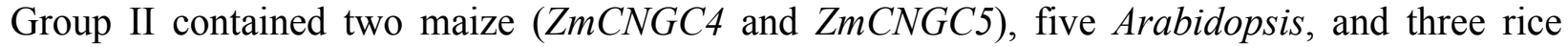
CNGC genes. Group III contained three maize CNGC genes (ZmCNGC6, ZmCNGC7, and ZmCNGC8); and Group IV contained five rice and four Arabidopsis CNGC genes. The maize genes in Group IV were separated into two groups, Group IVa contained $Z m C N G C 9$ and Group

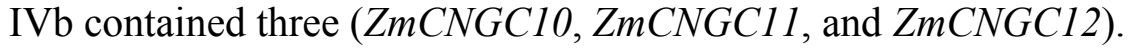

The phylogenetic tree also showed that the maize $C N G C$ genes could also be grouped into five groups (Figure 3A). Consistent with other plant $C N G C$ genes, Group IVa contained only one or two gene members and formed the smallest group (Saand et al. 2015b). The results suggested that 218 during the evolution of CNGCs, Group IV CNGC genes may have emerged first whereas Groups 
219 I and II $C N G C$ genes appeared later. Furthermore, the tree topology produced by the neighbor 220 joining analyses was the same as the ML tree in Figure 2, and it contained all the groups 221 (Supplemental File 3).

222 Additionally, only one segmental duplication gene pair, ZmCNGC10-ZmCNGC11, was formed in 223 the maize genome (Table 2). The evolutionary process between maize CNGCs and other 224 gramineae plants was further explored by investigating the genome synteny among Sorghum, rice, 225 and Brachypodium. The results showed that there were two, two and one $Z m C N G C$ genes that 226 showed syntenic bias towards particular Sorghum, rice, and Brachypodium chromosomes, 227 respectively (Table 2 ). In addition, $\mathrm{Ka} / \mathrm{Ks}$ was used to evaluate their specific positions under 228 positive selection pressure after duplication (Mayrose et al. 2007). $\mathrm{Ka} / \mathrm{Ks}$ values that $=1,<1$ or $>$ 2291 indicate neutral, purifying, and positive selection, respectively (Lynch and Conery 2000). The $230 \mathrm{Ka} / \mathrm{Ks}$ value of each gene pair was calculated and the $\mathrm{Ka} / \mathrm{Ks}$ value for all gene pairs was less than 231 1, which suggested that these genes had evolved under strong purifying selection. Furthermore, 232 the results indicated that the divergence of maize CNGCs from other gramineae plants did not 233 occur evenly.

\section{$Z m C N G C$ gene structures and the conserved motif analyses}

236

237

Gene structure analysis could improve our understanding of gene function and evolution. The number of introns ranged from 0 to 7 (Figure 3B), which was different from rice and Arabidopsis CNGCs. In rice, the OsCNGCs introns numbers ranged from 1 to 11, whereas Arabidopsis CNGCs ranged from 4 to 10 (Nawaz et al. 2014). The Group IVa and IVb ZmCNGCs had distinct gene structures compared to those of the other groups, with more introns at different phases and lengths, which is consistent with most flowering plant species Group IV CNGC genes (Saand et al. 2015b).

The motif-based recognition of proteins can improve understanding of their the evolutionary history (Seoighe and Gehring 2004). Ten putative motifs were characterized and named as motif1 to motif10 in the ZmCNGCs. The relative positions of the motifs in the five groups were found to 
246 have various patterns (Figure 3C and 3D), for example, all the ZmCNGCs contained motif1, 247 motif2, motif3, and motif4, which meant they presented typical ZmCNGC domains. Motif3 was a 248 combination of the calmodulin binding (CaMB) domain and the IQ domain 249 (QWRTWAA[CV]FIQ[AL]AW[RH]RY), and motif4 was the cyclic nucleotide-binding (CNB) 250 domain, which was located in the C-terminal. Furthermore, motif10, 9, 5, 8 (or 6), 2, and 1 were 251 the motif logos of the transmembrane domains. They represented the S1, S2, S3, S4, S5, and S6 252 regions of the transmembrane domain in the N-terminal. Motif7 represented the ion transport 253 protein (ITP) domain. In addition, 10 of the ZmCNGCs, but not ZmCNGC2 and ZmCNGC3,

254

255

256

257

258

259

260

261

262

263

264

265

266

267

268

269

270

271

272

possessed motif 5 and 6 that are associated with ion transport (Nawaz et al. 2014). Although other motifs have not been reported in plants or animals, they undertake important functions within the organism.

\section{Prediction of cis-acting regulatory elements and protein-protein interactions that involve ZmCNGC proteins}

Cis-acting regulatory elements are important molecular switches that are associated with the transcriptional regulation of genes when environmental stresses are encountered (Nakashima et al. 2009). To better understand the possible biological processes undertaken by the ZmCNGCs involved, $1.5 \mathrm{~kb}$ sequences upstream of the $Z m C N G C$ gene genomic sequences were used to identify cis-regulatory elements. A total of 137 different putative cis-elements were found to be associated with the identified $Z m C N G C$ genes and only 12, including CACTFTPPCA1, EBOXBNNAPA, DOFCOREZM, MYCCONSENSUSAT, CAATBOX1, GTGANTG10, WRKY71OS, GT1CONSENSUS, ROOTMOTIFTAPOX1, POLLEN1LELAT52, MYBCORE, and OSE2ROOTNODULE, were in the promoter region of the $Z m C N G C$ genes (Supplemental File 5: Table S2), which was highly consistent with rice CNGCs. Additionally, five cis-elements were gene-specific. ACGTCBOX, TATABOX3, CTRMCAMV35S, and HDZIP2ATATHB2 were unique to $Z m C N G C 6, Z m C N G C 7, Z m C N G C 8$, and $Z m C N G C 11$, respectively. Additionally, some cis-elements were involved in responses to different abiotic/biotic stimuli, including 
273 hormones (e.g. abscisic acid, auxin, ethylene), stress (e.g. drought, temperatures, disease) and 274 development (e.g. mesophyll specific, tissue specific) responses, which indicated that these $275 Z m C N G C$ genes might be involved in regulating diverse stress responses.

276 The ZmCNGC protein-protein interactions were predicted in order to gain a better understanding 277 of $\mathrm{ZmCNGC}$ protein roles in the plant. A total of 120 protein pairs were predicted to interact 278 between the $12 \mathrm{ZmCNGC}$ proteins and the other 11 maize proteins, and ZmCNGC8 was found to 279 interact with $\mathrm{ZmCNGC10}$ by prediction analysis (Figure 4 and Supplemental File 5: Table S3). 280 We also used genes in Arabidopsis that were homologous to the ZmCNGC genes to predict the 281 protein-protein interactions. The results showed that three were validated by the experimental data 282 (Supplemental File 5: Table S3).

283

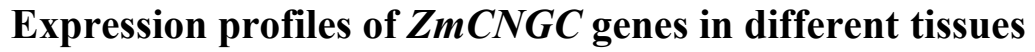

The physiological function of $Z m C N G C$ genes was investigated using transcriptome sequencing which evaluated the tissue-specific expression levels of $\mathrm{ZmCNGC}$ genes in different tissues (see Materials and Methods section). The expression levels among the $Z m C N G C$ genes are shown in Figure 5A and Supplemental File 5: Table S4 suggested that their expression levels were tissuespecific. For example, $Z m C N G C 5$ was specifically expressed in pollen compared to other tissues, which implied that it played a particular role in pollen physiological development, whereas

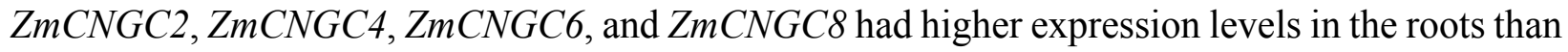
in the other tissues, which suggested that they had important roles in root growth and development. All Group IVb ZmCNGC genes, including ZmCNGC10, ZmCNGC11, and ZmCNGC12, were relatively highly expressed in the embryo, which implied that these genes played crucial roles in the growth and development of the maize embryo.

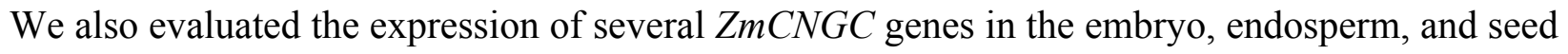
for several days after pollination. $Z m C N G C 3, Z m C N G C 5$ and $Z m C N G C 7$ were not detected or had no expression in any of the tissues. However, ZmCNGC5 was expressed in pollen (Fig. 5A, B, C, and D). Cis-acting regulatory elements analysis showed that only $Z m C N G C 5$ and $Z m C N G C 7$ did 
300

301

302

303

304

305

306

307

308

309

310

311

312

313

314

315

316

317

318

319

320

321

322

323

324

325

326

not contain CANBNNAPA, which is the element required for embryo and endosperm-specific transcription (Ellerström et al. 1996). This might be the reason why they did not show any

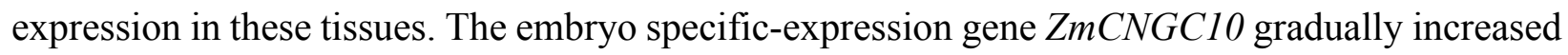
over time in the embryo (Figure 5B). $Z m C N G C 8$ was highly expressed in the embryo, endosperm, and seeds after pollination, and the Group IVb gene showed a similar expression pattern.

\section{DISCUSSION}

\section{Features and evolution of plant CNGC family genes}

Plant CNGC family genes are characterized by the presence of a CNBD in the C-terminal and a hexa-TM in the N-terminal (Saand et al. 2015b). A total of $12 \mathrm{ZmCNGC}$ genes were identified after a BLAST search against the maize genome protein sequences. Among them, ZmCNGC3 showed no ESTs and was not expressed in all the tissues, which suggested that it was a nonexpressed pseudogene. In Arabidopsis, AtCNGC16 (AT3G48010) had no EST alignments and might also be a pseudogene (Mäser et al. 2001). Many other ion transporters also possess these domains. For example, potassium AKT/KAT channels (Shaker type) contain both a CNBD domain and a TM domain. All AKT/ KAT-type channels consist of six transmembrane (TM) regions with one $\mathrm{P}$ region ( $\mathrm{Su}$ et al. 2001). The $\mathrm{ML}$ phylogenetic tree showed that the maize CNGC and the AKT/KAT-type channel genes were clustered into two separate sections (Supplemental File 1). Previous studies have shown that the CNGC-specific motif with a PBC and a hinge domain (LX(2)-G-[ED]-ELL-[TSG]-W-[ACY]-L-X(10,20)-[SA]-X-T-X(7)-[EQ]-[AG]-F-X-L) only exists in maize plant CNGCs and does not occur in the ion transporters found in other plants, such as rice, Arabidopsis, and tomato (Saand et al. 2015b; Nawaz et al. 2014; Zelman et al. 2013). In this study, $12 \mathrm{ZmCNGCs}$ were identified and the PBCs and hinge domains were highly conserved after alignment, which further confirmed the previous hypothesis (Saand et al. 2015b). The conserved motif analysis showed that the motif3 (QWRTWAA[CV]FIQ[AL]AW[RH]RY) pattern was the IQ domain among these $12 \mathrm{ZmCNGCs.} \mathrm{The} \mathrm{IQ} \mathrm{domain} \mathrm{is} \mathrm{conserved} \mathrm{among} \mathrm{plant} \mathrm{CNGCs} \mathrm{and}$ enhances the changeable $\mathrm{Ca}^{2+}$-dependent channel control mechanisms in plants (Fischer et al. 
327

328

329

330

331

332

333

334

335

336

337

338

339

340

341

342

343

344

345

346

347

348

349

350

351

352

353

2013). The results from this study showed that all CNGC proteins contained the IQ motif, which suggested that they bind $\mathrm{CaM}$ in a $\mathrm{Ca}^{2+}$-dependent manner. Notably, motif4 is the sequence logo of the CNBD domain, which is conserved in most plants and animals (Jackson et al. 2007).

The results showed that the CNGC family in 12 gramineae plants at various evolutionary nodes is adequate for analyzing the phylogeny and evolution of the CNGC gene family in gramineae plants. In Supplemental File 5, Table 1 shows that 4 out of 12 plants under analysis contained $<20$ CNGC genes. These were $B$. distachyon (16), O. sativa (16), S. bicolor (13) and maize (12). The ML tree for the 273 CNGCs clearly indicated that gramineae CNGCs clustered into six groups (I, II, III, IV, IVa, and IVb) with significant bootstrap values; Groups IV, IVa and IVb had particularly high bootstrap support values, but group IV CNGCs did not cluster with B. distachyon, O. sativa, $S$. bicolor, and maize CNGCs. This suggested that there are missing CNGCs that need to be identified or there was duplication during evolution. Davidson et al. (2013) showed that gene duplication can lead to the generation of significant numbers of novel genes. Therefore, these results imply that duplication events play a principal role in gene evolution. Phylogenetic, gene structure, and

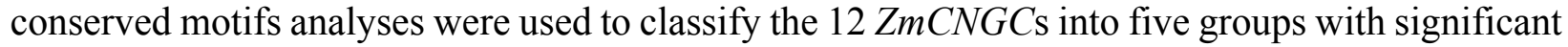

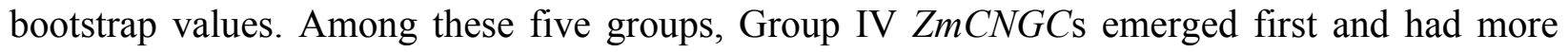
introns than the genes in the other groups.

\section{$C N G C$ genes play an important role in plant development}

The CNGCs are involved in the regulation of plant growth and development (Chin et al. 2009b). This study focused on the role that maize CNGCs play in different tissues by investigating its expression in plant embryo, endosperm and seed expression. The results showed that some $\mathrm{ZmCNGC}$ genes have tissue-specific expression. For example, ZmCNGC2, ZmCNGC4, $\mathrm{ZmCNGC6}$ and $\mathrm{ZmCNGC8}$ were highly expressed in the roots; ZmCNGC5 was specifically expressed in pollen; and all Group IVb genes were expressed in the embryo. Group IVb genes are the evolutionary ancestors of CNGC genes and were mainly expressed in the embryo and seeds after pollination, which suggested that Group IVb genes play a significant role in embryo 
354 development. In addition, all Group IVb genes were linked to gene duplication and they had a 355 similar expression pattern in the different tissues, which indicated that their function was to 356 enhance adaptability during gene evolution.

357 Most previous studies have shown that $C N G C$ genes are related to pollen development and 358 responses to environmental stimuli. For example, Arabidopsis CNGC16 is critical for pollen 359 fertility under heat and drought stress (Tunc-Ozdemir et al. 2013b), and $C N G C 18$ has been shown 360 to play a role in pollen tube tip growth (Frietsch et al. 2007). In rice, OsCNGC13 promotes the 361 seed-setting rate by facilitating pollen tube growth in stylar tissues (Xu et al. 2017). ZmCNGC1

362

363

364

365

366

367

368

369

370

371

372

373

374

375

376

377

378

379

380 and $\mathrm{ZmCNGC5}$, two homologous genes of $C N G C 16$ and $C N G C 18$, are mainly expressed in pollen, which indicates that they are predominantly involved in pollen development. Previous studies have shown that $A t C N G C 3$ is mainly expressed in the embryo, leaves, and roots, and $\mathrm{ZmCNGC4}$ expression level is similar to that of $A t C N G C 3$ which was highly expressed during plant development (Kaplan et al. 2007). These results imply that these genes play crucial roles during the growth and development of maize.

\section{CONCLUSION}

A total of 12 CNGC genes were identified in maize via bioinformatics. The results were based on the presence of a plant CNGC-specific motif that spanned the PBCs and hinge domain in the CNBD of CNGC proteins. Phylogenetic analyses showed that Group IV ZmCNGCs emerged first and had more introns than the other ZmCNGC groups, whereas Groups I and II evolved later. Phylogenetic analysis of 12 gramineae plants revealed that some CNGCs are probably missing or have been duplicated during evolution. Significantly, the $Z m C N G C$ genes had diversity in gene structures, protein lengths and sizes. We modified a maize stringent motif (L-X(2)-G-[ED]-ELL[TSG]-W-[ACY]-L-X(10,20)-[SA]-X-T-X(7)-[EQ]-[AG]-F-X-L) that contained a PBC and a hinge domain to better characterize classification. Expression profiles of the $Z m C N G C$ genes were tissue-specific and were related to pollen development. This study provides important information about plant CNGCs during gene evolution. 


\section{ACKNOWLEDGEMENTS}

383 We extend our gratitude to Dr. Lihe $\mathrm{Yu}$ for providing valuable suggestions.

\section{ADDITIONAL INFORMATION AND DECLARATIONS}

387

388

389

390

391

392

393

394

395

396

397

398

399

400

401

402

403

404

405

406

407

\section{Supplemental Information}

\section{Supplemental File 1}

A ML phylogenetic tree, conserved regions and multiple sequences alignments between maize AKT/KAT channels genes and ZmCNGCs.

Supplemental File 2

Multiple sequences alignments among maize, Arabidopsis and rice CNGCs by using T-COFFEE method.

Supplemental File 3

The un-rooted neighbor joining (NJ) tree based on the maize, rice and Arabidopsis CNGCs protein sequences by using MEGA7.

\section{Supplemental File 4}

The Maximum likelihood (ML) tree based on the maize and other 11 gramineous plants CNGCs protein sequences by using IQTREE.

Supplemental File 5

Table S1 The GNGC information of 12 gramineae plants

Table S2 Numbers of known stress-related elements in the promoter regions of ZmCNGCs

Table S3 The protein-protein interaction information of maize CNGC protein with other maize proteins

04 Table S4 The FPKM data of $Z m C N G C$ genes in different tissues.

Supplemental File 6

406 The logo of ten motifs in study.

07 Supplemental File 7 
408 The gene sequences used in this research.

409 Data Availability

410 The following information was supplied regarding data availability:

411 The raw data has been supplied as a Supplemental File 7.

412

\section{REFERENCES}

414

415

416

417

418

419

420

421

422

423

424

425

426

427

428

429

430

431

432

433

434

435

436

437

438

439

440

441

442

443

444

445

Ali R, Ma W, Lemtiri-Chlieh F, Tsaltas D, Leng Q, Bodman SV, Berkowitz GA (2007) Death Don't Have No Mercy and Neither Does Calcium: Arabidopsis CYCLIC NUCLEOTIDE GATED CHANNEL2 and Innate Immunity. The Plant cell 19 (3):1081-1095

Artimo P, Jonnalagedda M, Arnold K, Baratin D, Csardi G, de Castro E, Duvaud S, Flegel V, Fortier A, Gasteiger E, Grosdidier A, Hernandez C, Ioannidis V, Kuznetsov D, Liechti R, Moretti S, Mostaguir K, Redaschi N, Rossier G, Xenarios I, Stockinger H (2012) ExPASy: SIB bioinformatics resource portal. Nucleic Acids Res 40 (Web Server issue):W597-603. doi:10.1093/nar/gks400

Bailey TL, Johnson J, Grant CE, Noble WS (2015) The MEME Suite. Nucleic Acids Res 43 (W1):W39-49. doi:10.1093/nar/gkv416

Balague C (2003) HLM1, an Essential Signaling Component in the Hypersensitive Response, Is a Member of the Cyclic Nucleotide-Gated Channel Ion Channel Family. The Plant Cell Online 15 (2):365-379. doi:10.1105/tpc.006999

Bolser D, Staines DM, Pritchard E, Kersey P (2016) Ensembl Plants: Integrating Tools for Visualizing, Mining, and Analyzing Plant Genomics Data. Methods in molecular biology (Clifton, NJ) 1374:115-140. doi:10.1007/978-1-4939-3167-5_6

Borsics T, Webb D, Andemeondzighi C, Staehelin LA, Christopher DA (2007) The cyclic nucleotide-gated calmodulin-binding channel AtCNGC10 localizes to the plasma membrane and influences numerous growth responses and starch accumulation in Arabidopsis thaliana. Planta 225 (3):563-573. doi:10.1007/s00425-006$0372-3$

Bridges D, Fraser ME, Moorhead GB (2005) Cyclic nucleotide binding proteins in the Arabidopsis thaliana and Oryza sativa genomes. Bmc Bioinformatics 6 (1):1-12. doi:10.1186/1471-2105-6-6

Chérel I (2004) Regulation of $\mathrm{K}+$ channel activities in plants: from physiological to molecular aspects. Journal of Experimental Botany 55 (396):337-351

Chen J, Yin H, Gu J, Li L, Liu Z, Jiang X, Zhou H, Wei S, Zhang S, Wu J (2015) Genomic characterization, phylogenetic comparison and differential expression of the cyclic nucleotide-gated channels gene family in pear (Pyrus bretchneideri Rehd.). Genomics 105 (1):39. doi:10.1016/j.ygeno.2014.11.006

Chin K, Moeder W, Yoshioka K (2009a) Biological roles of cyclic-nucleotide-gated ion channels in plants: what we know and don't know about this 20 member ion channel family. Botany-botanique 87 (7):668-677. doi:10.1139/B08-147

Chin KC, Moeder WM, Yoshioka KY (2009b) Biological roles of cyclic-nucleotide-gated ion channels in plants: What we know and don't know about this 20 member ion channel family. Botany-botanique 87 (7):668-677

Davidson CJ, Guthrie EE, Lipsick JS (2013) Duplication and maintenance of the Myb genes of vertebrate animals. 
Biology Open 2 (2):101-110

Defalco TA, Marshall CB, Munro K, Kang HG, Moeder W, Ikura M, Snedden WA, Yoshioka K (2016) Multiple Calmodulin-Binding Sites Positively and Negatively Regulate Arabidopsis CYCLIC NUCLEOTIDEGATED CHANNEL12. Plant Cell 28 (7):1738. doi:10.1105/tpc.15.00870

Di Tommaso P, Moretti S, Xenarios I, Orobitg M, Montanyola A, Chang JM, Taly JF, Notredame C (2011) T-Coffee: a web server for the multiple sequence alignment of protein and RNA sequences using structural information and homology extension. Nucleic Acids Res 39 (Web Server issue):W13-17. doi:10.1093/nar/gkr245

Diller TC, Madhusudan, Xuong NH, Taylor SS (2001) Molecular Basis for Regulatory Subunit Diversity in cAMPDependent Protein Kinase - Crystal Structure of the Type IIbeta Regulatory Subunit. Structure 9 (1):73-82. doi:10.1016/S0969-2126(00)00556-6

Ellerström M, Stålberg K, Ezcurra I, Rask L (1996) Functional dissection of a napin gene promoter: identification of promoter elements required for embryo and endosperm-specific transcription. Plant Molecular Biology 32 (6):1019-1027

Fischer C, Kugler A, Hoth S, Dietrich P (2013) An IQ Domain Mediates the Interaction with Calmodulin in a Plant Cyclic Nucleotide-Gated Channel. Plant and Cell Physiology 54 (4):573-584. doi:10.1093/pcp/pct021

Frietsch S, Wang YF, Sladek C, Poulsen LR, Romanowsky SM, Schroeder JI, Harper JF (2007) A cyclic nucleotidegated channel is essential for polarized tip growth of pollen. Proc Natl Acad Sci U S A 104 (36):14531-14536

Gao F, Han X, Wu J, Zheng S, Shang Z, Sun D, Zhou R, Li B (2012) A heat-activated calcium-permeable channel-Arabidopsis cyclic nucleotide-gated ion channel 6--is involved in heat shock responses. Plant Journal for Cell \& Molecular Biology 70 (6):1056-1069

Gao QF, Gu LL, Wang HQ, Fei CF, Fang X, Hussain J, Sun SJ, Dong JY, Liu H, Wang YF (2016) Cyclic nucleotidegated channel 18 is an essential $\mathrm{Ca} 2+$ channel in pollen tube tips for pollen tube guidance to ovules in Arabidopsis. Proc Natl Acad Sci U S A 113 (11):3096-3101

Guo S, Zuo Y, Zhang Y, Wu C, Su W, Jin W, Yu H, An Y, Li Q (2017) Large-scale transcriptome comparison of sunflower genes responsive to Verticillium dahliae. Bmc Genomics 18 (1):42. doi:10.1186/s12864-016$3386-7$

He Z, Zhang H, Gao S, Lercher MJ, Chen WH, Hu S (2016) Evolview v2: an online visualization and management tool for customized and annotated phylogenetic trees. Nucleic Acids Research 44 (Web Server issue):W236W241

Higo K, Ugawa Y, Iwamoto M, Korenaga T (1999) Plant cis-acting regulatory DNA elements (PLACE) database: 1999. Nucleic Acids Res 27 (1):297-300

Hu B, Jin J, Guo AY, Zhang H, Luo J, Gao G (2015) GSDS 2.0: an upgraded gene feature visualization server. Bioinformatics (Oxford, England) 31 (8):1296-1297. doi:10.1093/bioinformatics/btu817

Hua BG, Mercier RW, Zielinski RE, Berkowitz GA (2003) Functional interaction of calmodulin with a plant cyclic nucleotide gated cation channel. Plant Physiology \& Biochemistry 41 (11-12):945-954

Jackson HA, Marshall CR, Accili EA (2007) Evolution and structural diversification of hyperpolarization-activated cyclic nucleotide-gated channel genes. Physiological Genomics 29 (3):231

Köhler C, Neuhaus G (2000) Characterisation of calmodulin binding to cyclic nucleotide-gated ion channels from Arabidopsis thaliana. FEBS letters 471 (2-3):133-136. doi:10.1016/S0014-5793(00)01383-1

Kakar KU, Nawaz Z, Kakar K, Ali E, Almoneafy AA, Ullah R, Ren XL, Shu QY (2017) Comprehensive genomic analysis of theCNGCgene family inBrassica oleracea: novel insights into synteny, structures, and transcript 
profiles. Bmc Genomics 18 (1):869. doi:10.1186/s12864-017-4244-y

Kaplan B, Sherman T, Fromm H (2007) Cyclic nucleotide-gated channels in plants. FEBS letters 581 (12):2237-2246. doi:10.1016/j.febslet.2007.02.017

Kugler A, Köhler B, Palme K, Wolff P, Dietrich P (2009) Salt-dependent regulation of a CNG channel subfamily in Arabidopsis. BMC Plant Biology 9 (1):140. doi:10.1186/1471-2229-9-140

Kumar S, Stecher G, Tamura K (2016) MEGA7: Molecular Evolutionary Genetics Analysis Version 7.0 for Bigger Datasets. Molecular Biology \& Evolution 33 (7):1870. doi:10.1093/molbev/msw054

Lam-Tung N, Schmidt HA, Arndt VH, Quang MB (2015) IQ-TREE: A Fast and Effective Stochastic Algorithm for Estimating Maximum-Likelihood Phylogenies. Molecular Biology \& Evolution 32 (1):268-274

Lee TH, Tang H, Wang X, Paterson AH (2013) PGDD: a database of gene and genome duplication in plants. Nucleic Acids Research 41 (Database issue):1152-1158

Lemtiri-Chlieh F, Berkowitz GA (2004) Cyclic adenosine monophosphate regulates calcium channels in the plasma membrane of Arabidopsis leaf guard and mesophyll cells. The Journal of biological chemistry 279 (34):35306-35312. doi:10.1074/jbc.M400311200

Letunic I, Bork P (2018) 20 years of the SMART protein domain annotation resource. Nucleic Acids Res 46 (D1):D493-d496. doi:10.1093/nar/gkx922

Librado P, Rozas J (2009) DnaSP v5: a software for comprehensive analysis of DNA polymorphism data. Bioinformatics (Oxford, England) 25 (11):1451

Lu M, Zhang Y, Tang S, Pan J, Yu Y, Han J, Li Y, Du X, Nan Z, Sun Q (2015) AtCNGC2 is involved in jasmonic acid-induced calcium mobilization. Journal of Experimental Botany 67 (3):809

Lynch M, Conery JS (2000) The evolutionary fate and consequences of duplicate genes. Science (New York, NY) 290 (5494): 1151

Mäser P, Thomine S, Schroeder JI, Ward JM, Hirschi K, Sze H, Talke IN, Amtmann A, Maathuis FJ, Sanders D (2001) Phylogenetic relationships within cation transporter families of Arabidopsis. Plant physiology 126 (4):1646-1667

Marchler-Bauer A, Bo Y, Han L, He J, Lanczycki CJ, Lu S, Chitsaz F, Derbyshire MK, Geer RC, Gonzales NR, Gwadz M, Hurwitz DI, Lu F, Marchler GH, Song JS, Thanki N, Wang Z, Yamashita RA, Zhang D, Zheng C, Geer LY, Bryant SH (2017) CDD/SPARCLE: functional classification of proteins via subfamily domain architectures. Nucleic Acids Res 45 (D1):D200-d203. doi:10.1093/nar/gkw1129

Maser P, Thomine S, Schroeder JI, Ward JM, Hirschi K, Sze H, Talke IN, Amtmann A, Maathuis FJ, Sanders D, Harper JF, Tchieu J, Gribskov M, Persans MW, Salt DE, Kim SA, Guerinot ML (2001) Phylogenetic relationships within cation transporter families of Arabidopsis. Plant physiology 126 (4):1646-1667

Mayrose I, Doron-Faigenboim A, Bacharach E, Pupko T (2007) Towards realistic codon models: among site variability and dependency of synonymous and non-synonymous rates. Bioinformatics (Oxford, England) 23 (13):i319-327. doi:10.1093/bioinformatics/btm176

Nakashima K, Ito Y, Yamaguchi-Shinozaki K (2009) Transcriptional regulatory networks in response to abiotic stresses in Arabidopsis and grasses. Plant physiology 149 (1):88-95. doi:10.1104/pp.108.129791

Nawaz Z, Kakar KU, Saand MA, Shu QY (2014) Cyclic nucleotide-gated ion channel gene family in rice, identification, characterization and experimental analysis of expression response to plant hormones, biotic and abiotic stresses. BMC Genomics 15 (1):1-18

Paterson AH, Bowers JE, Chapman BA (2004) Ancient polyploidization predating divergence of the cereals, and its 
consequences for comparative genomics. Proceedings of the National Academy of Sciences of the United States of America 101 (26):9903-9908. doi:10.1073/pnas.0307901101

Robert X, Gouet P (2014) Deciphering key features in protein structures with the new ENDscript server. Nucleic Acids Research 42 (Web Server issue):W320. doi:10.1093/nar/gku316

Saand MA, Xu YP, Li W, Wang JP, Cai XZ (2015a) Cyclic nucleotide gated channel gene family in tomato: genomewide identification and functional analyses in disease resistance. Frontiers in Plant Science 6 (303):303. doi:10.3389/fpls.2015.00303

Saand MA, Xu YP, Munyampundu JP, Li W, Zhang XR, Cai XZ (2015b) Phylogeny and evolution of plant cyclic nucleotide-gated ion channel (CNGC) gene family and functional analyses of tomato CNGCs. DNA research : an international journal for rapid publication of reports on genes and genomes 22 (6):471-483

Schuurink RC, Shartzer SF, Fath A, Jones RL (1998) Characterization of a calmodulin-binding transporter from the plasma membrane of barley aleurone. Proceedings of the National Academy of Sciences of the United States of America 95 (4):1944

Seoighe C, Gehring C (2004) Genome duplication led to highly selective expansion of the Arabidopsis thaliana proteome. Trends in genetics : TIG 20 (10):461-464. doi:10.1016/j.tig.2004.07.008

Shannon P, Markiel A, Ozier O, Baliga NS, Wang JT, Ramage D, Amin N, Schwikowski B, Ideker T (2003) Cytoscape: A Software Environment for Integrated Models of Biomolecular Interaction Networks. Genome Res 13 (11):2498-2504. doi:10.1101/gr.1239303

Su H, Golldack D, Katsuhara M, Zhao C, Bohnert HJ (2001) Expression and Stress-Dependent Induction of Potassium Channel Transcripts in the Common Ice Plant. Plant physiology 125 (2):604-614

Szklarczyk D, Morris JH, Cook H, Kuhn M, Wyder S, Simonovic M, Santos A, Doncheva NT, Roth A, Bork P (2017) The STRING database in 2017: quality-controlled protein-protein association networks, made broadly accessible. Nucleic Acids Research 45 (Database issue):D362-D368

Talke, Ina N, Blaudez, Damien, Maathuis, Frans JM, Sanders, Dale (2003) CNGCs: prime targets of plant cyclic nucleotide signalling? Trends in Plant Science 8 (6):286. doi:10.1016/S1360-1385(03)00099-2

Tunc-Ozdemir M, Rato C, Brown E, Rogers S, Mooneyham A, Frietsch S, Myers CT, Poulsen LR, Rui M, Harper JF (2013a) Cyclic Nucleotide Gated Channels 7 and 8 Are Essential for Male Reproductive Fertility. Plos One 8 (2):e55277

Tunc-Ozdemir M, Tang C, Ishka MR, Brown E, Groves NR, Myers CT, Rato C, Poulsen LR, Mcdowell S, Miller G (2013b) A cyclic nucleotide-gated channel (CNGC16) in pollen is critical for stress tolerance in pollen reproductive development. Plant physiology 161 (2):1010-1020. doi:10.1104/pp.112.206888

Wang Y, Kang Y, Ma C, Miao R, Wu C, Long Y, Ge T, Wu Z, Hou X, Zhang J (2017) CNGC2 Is a Ca2+ Influx Channel That Prevents Accumulation of Apoplastic Ca2+ in the Leaf. Plant physiology 173 (2):1342-1354

Ward JM, Mäser P, Schroeder JI (2009) Plant Ion Channels: Gene Families, Physiology, and Functional Genomics Analyses. Annual Review of Physiology 71 (71):59. doi:10.1146/annurev.physiol.010908.163204

Xu Y, Yang J, Wang Y, Wang J, Yu Y, Long Y, Wang Y, Zhang H, Ren Y, Chen J, Wang Y, Zhang X, Guo X, Wu F, Zhu S, Lin Q, Jiang L, Wu C, Wang H, Wan J (2017) OsCNGC13 promotes seed-setting rate by facilitating pollen tube growth in stylar tissues. PLoS genetics 13 (7):e1006906. doi:10.1371/journal.pgen.1006906

Yu CS, Lin CJ, Hwang JK (2010) Predicting subcellular localization of proteins for Gram-negative bacteria by support vector machines based on n-peptide compositions. Protein Science 13 (5):1402-1406

Yu J, Wang J, Lin W, Li S, Li H, Zhou J, Ni P, Dong W, Hu S, Zeng C, Zhang J, Zhang Y, Li R, Xu Z, Li S, Li X, 
569

570

571

572

573

574

575

576

577

578

579

580

581

582

583

584

585

586
Zheng H, Cong L, Lin L, Yin J, Geng J, Li G, Shi J, Liu J, Lv H, Li J, Wang J, Deng Y, Ran L, Shi X, Wang X, Wu Q, Li C, Ren X, Wang J, Wang X, Li D, Liu D, Zhang X, Ji Z, Zhao W, Sun Y, Zhang Z, Bao J, Han Y, Dong L, Ji J, Chen P, Wu S, Liu J, Xiao Y, Bu D, Tan J, Yang L, Ye C, Zhang J, Xu J, Zhou Y, Yu Y, Zhang B, Zhuang S, Wei H, Liu B, Lei M, Yu H, Li Y, Xu H, Wei S, He X, Fang L, Zhang Z, Zhang Y, Huang X, Su Z, Tong W, Li J, Tong Z, Li S, Ye J, Wang L, Fang L, Lei T, Chen C, Chen H, Xu Z, Li H, Huang H, Zhang F, Xu H, Li N, Zhao C, Li S, Dong L, Huang Y, Li L, Xi Y, Qi Q, Li W, Zhang B, Hu W, Zhang Y, Tian X, Jiao Y, Liang X, Jin J, Gao L, Zheng W, Hao B, Liu S, Wang W, Yuan L, Cao M, McDermott J, Samudrala R, Wang J, Wong GK, Yang H (2005) The Genomes of Oryza sativa: a history of duplications. PLoS biology 3 (2):e38. doi:10.1371/journal.pbio.0030038

Yuen CCY, Christopher DA (2013) The group IV-A cyclic nucleotide-gated channels, CNGC19 and CNGC20, localize to the vacuole membrane in Arabidopsis thaliana. AoB Plants 5:plt012-plt012. doi:10.1093/aobpla/plt012

Zelman AK, Dawe A, Berkowitz GA (2013) Identification of cyclic nucleotide gated channels using regular expressions. Methods in Molecular Biology 1016:207

Zelman AK, Dawe A, Gehring C, Berkowitz GA (2012) Evolutionary and Structural Perspectives of Plant Cyclic Nucleotide-Gated Cation Channels. Frontiers in Plant Science 3:95 
Table $\mathbf{1}$ (on next page)

Characteristic features of $Z m C N G C$ genes in maize 
1 Table 1 Characteristic features of $Z m C N G C$ genes in maize

\begin{tabular}{|c|c|c|c|c|c|c|c|c|c|c|c|}
\hline Group & Gene ID & Gene name & $\mathrm{Chr}^{[1]}$ & $\operatorname{Start}^{[2]}$ & end ${ }^{[3]}$ & $\begin{array}{l}\text { Length } \\
(\text { aa })^{[4]}\end{array}$ & $\begin{array}{c}\text { MW } \\
(\mathrm{Da})^{[5]}\end{array}$ & $\mathrm{pI}^{[6]}$ & GRAVY[7] & Localization $^{[8]}$ & $\mathrm{EST}^{[9]}$ \\
\hline \multirow[t]{3}{*}{ I } & $\mathrm{ZmCNGC1}$ & GRMZM2G148118 & 4 & 230194909 & 230205383 & 701 & 79914.66 & 9.18 & -0.046 & PlasmaMembrane & 17 \\
\hline & $\mathrm{ZmCNGC2}$ & GRMZM2G129375 & 6 & 109824359 & 109825921 & 626 & 38405.22 & 9.17 & -0.484 & PlasmaMembrane & 23 \\
\hline & ZmCNGC3 & GRMZM2G066269 & 4 & 230382717 & 230384596 & 329 & 38632.18 & 9.59 & -0.52 & Nuclear & 0 \\
\hline \multirow[t]{2}{*}{ II } & ZmCNGC4 & GRMZM2G023037 & 2 & 5966501 & 5978989 & 723 & 82856.52 & 8.92 & -0.114 & PlasmaMembrane & 29 \\
\hline & ZmCNGC5 & GRMZM2G077828 & 5 & 17453069 & 17455739 & 699 & 80110.81 & 9.42 & -0.091 & PlasmaMembrane & 15 \\
\hline \multirow[t]{3}{*}{ III } & ZmCNGC6 & GRMZM2G005791 & 5 & 218834435 & 218840917 & 700 & 80057.39 & 8.97 & -0.078 & PlasmaMembrane & 31 \\
\hline & $\mathrm{ZmCNGC7}$ & GRMZM2G068904 & 5 & 191609046 & 191611784 & 689 & 80038.95 & 9.8 & -0.117 & PlasmaMembrane & 15 \\
\hline & ZmCNGC8 & GRMZM2G135651 & 7 & 150652512 & 150657060 & 739 & 85523.26 & 9.33 & -0.148 & PlasmaMembrane & 61 \\
\hline IVa & ZmCNGC9 & GRMZM2G141642 & 5 & 217119224 & 217125465 & 463 & 53303.64 & 9.48 & -0.059 & PlasmaMembrane & 13 \\
\hline \multirow[t]{3}{*}{$\mathrm{IVb}$} & $\mathrm{ZmCNGC10}$ & GRMZM5G858887 & 5 & 6938133 & 6943695 & 745 & 83672.62 & 9.46 & 0.081 & PlasmaMembrane & 84 \\
\hline & ZmCNGC11 & GRMZM2G074317 & 1 & 283401507 & 283408034 & 730 & 81440.82 & 9.43 & 0.067 & PlasmaMembrane & 74 \\
\hline & $\mathrm{ZmCNGC} 12$ & GRMZM2G090528 & 8 & 177244867 & 177247281 & 505 & 57042.47 & 9.75 & 0.014 & PlasmaMembrane & 48 \\
\hline
\end{tabular}

2 Note: [1] chromosome location; [2] star of gene location; [3] end of gene location; [4] the length

3 of ZmCNGC proteins; [5] the molecular weight of ZmCNGC proteins; [6] the Grand average of

4 hydropathicity of ZmCNGC proteins; [8] the prediction the ZmCNGCs subcellular location; [9]

5 expressed sequence tags blast results.

6 


\section{Table 2 (on next page)}

The $\mathrm{Ka} / \mathrm{Ks}$ ratios and estimated divergence time for orthologous CNGC proteins between maize and other Gramineae plants 
1 Table 2

$2 \mathrm{The} \mathrm{Ka} / \mathrm{Ks}$ ratios and estimated divergence time for orthologous CNGC proteins between maize and other

3 Gramineae plants

4

\begin{tabular}{cccccc}
\hline Gene ID & Gene ID & Ka & Ks & Ka/Ks & Mya \\
\hline ZmCNGC11 & ZmCNGC10 & 0.0258 & 0.1829 & 0.141061 & 14.06923 \\
ZmCNGC5 & Sobic.001G155100 & 0.5298 & 0.8917 & 0.594146 & 68.59231 \\
ZmCNGC12 & Sobic.009G188800 & 0.095 & 0.4878 & 0.194752 & 37.52308 \\
ZmCNGC12 & Sobic.003G317700 & 0.0306 & 0.0993 & 0.308157 & 7.638462 \\
ZmCNGC5 & LOC_Os03g44440 & 0.7415 & 1.2253 & 0.605158 & 94.25385 \\
ZmCNGC12 & LOC_Os05g42250 & 0.1187 & 0.4278 & 0.277466 & 32.90769 \\
ZmCNGC12 & LOC_Os01g57370 & 0.073 & 0.3017 & 0.241962 & 23.20769 \\
ZmCNGC5 & Bradi1g13740 & 0.7912 & 1.1721 & 0.675028 & 90.16154
\end{tabular}

5

6

7 
Figure 1 (on next page)

The maize CNGC-specific motif spans the putative PBC and the hinge within the CNBD of the $12 \mathrm{ZmCNGCs.}$

The diagram at the top represents three regions of plant CNGCs: the six transmembrane domains (TM), a CNBD containing a PBC and the hinge, and CaMBD. The maize CNGC-specific amino acid motif is shown below the cartoon. The asterisk indicate $100 \%$ identity among the $12 \mathrm{ZmCNGCs}$. Below is the alignment of CNBD domains of $12 \mathrm{ZmCNGCs}$. Residues in blue and yellow highlighted indicate the PBC and hinge domain, respectively. 

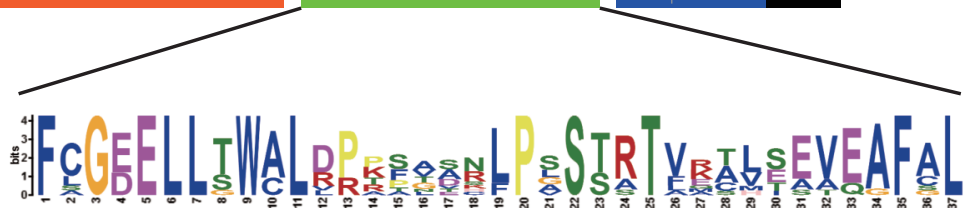

10

20

30

40

50

60

70

80

90

ZmCNGC1 ZmCNGC2

ZmCNGC3

ZmCNGC4

ZmCNGC5

ZmCNGC 6

ZmCNGC7

ZmCNGC8

ZmCNGC9

ZmCNGC10

$\mathrm{ZmCNGC} 11$

ZmCNGC12

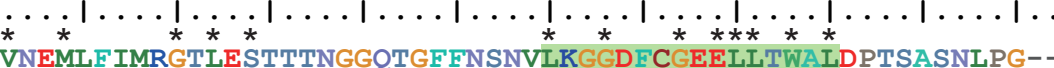

$1 \ldots$
1. VNEMLFVMRGNLMSMTTNGGRTGF FNSDVLKAGDE CGEELLTWALDPTSTSSLPS--- - - ---STRTVKTMSEVEAFALRAEDLRFVATQFRR VNEMFFIMRGRLESTTTDGGRAGFFNSNVLEGGDFCGEELLTWALDPASGSNLPS---------STRTARTLSEVEGFSLRARHLRFVASQYRR VDEMHFILHGCLESVTTDGGRSGF FNKVQLKEGS C GDELLTWALDPKSAANF'PV----------SSRTVQALTEVEAFALCAEELKFVASQFRR VDQMVFIIRGSLESITTDGGRTGFYNRSLLEEGDFCGEELLTWALDPKAGACLPS---------STRTVMALSEVEAFALHAEELKFVAGQFRR VTEMLFIIRGKLESSTTNGGRTGFFNSITLKPGD C GEELLGWALVPRPTTNLPS----------STRTVKALIEVEAFALQAEDLKFVASQFRR VDSMLFIIRGYLDSYTTQGGRSGF FNSCRIGAGE E GEELLTWALDPRPAAKLPL-- - - - - STRTVRAVSEVEAFALVADDLRFVASQFRR VNEMLFI IRGKLESSTTNGGRSNF FNSI ILRPGDFAGEELLTWALLPKTNVHFPL---------STRTVRSHTEVEAFALRAEDLKFVANQFRR VEKMVF IVRGKLESISADGSKA------PLHDGDVCGEELITWYLEHSSANRDGGKIKFOGMRLVAIRTVRCLTNVEAFVLRASDLEEVTSQFAR VQRMVF ILQGKLRS--TQPLTKGVVATCMLGAGNELGDELLSWCLRRPFVDRLPA---------SSATFECVEAAQAFCLDAPDLRFITEHFRY VQRMVFILQGKLRS--TQPLTKGVVATCMLGAGSFLGDELLSWCLRRPFVDRLPA---------SSATFECVEAAQAFCLGAPDLRFITEHFRY VKRMLF IVRGHLQS--SQVLRNGAESCCMLGPGNESGDELLSWCLRRPFLERLPG---------SSSTLATLESTEAFGLDAADVKYVTQHFRY

PBC $\quad \overline{\text { Hinge }}$




\section{Figure 2 (on next page)}

Phylogenetic relationships among the ZmCNGCs, OsCNGCs and AtCNGCs.

The multiple alignment was performed by ClustalX program. MEGA 7.0 was used to create maximum likelihood (ML) under the Jones-Taylor-Thornton (JTT) model. The tree was constructed with1000 bootstrap replications. Each group identified is indicated on the right. 


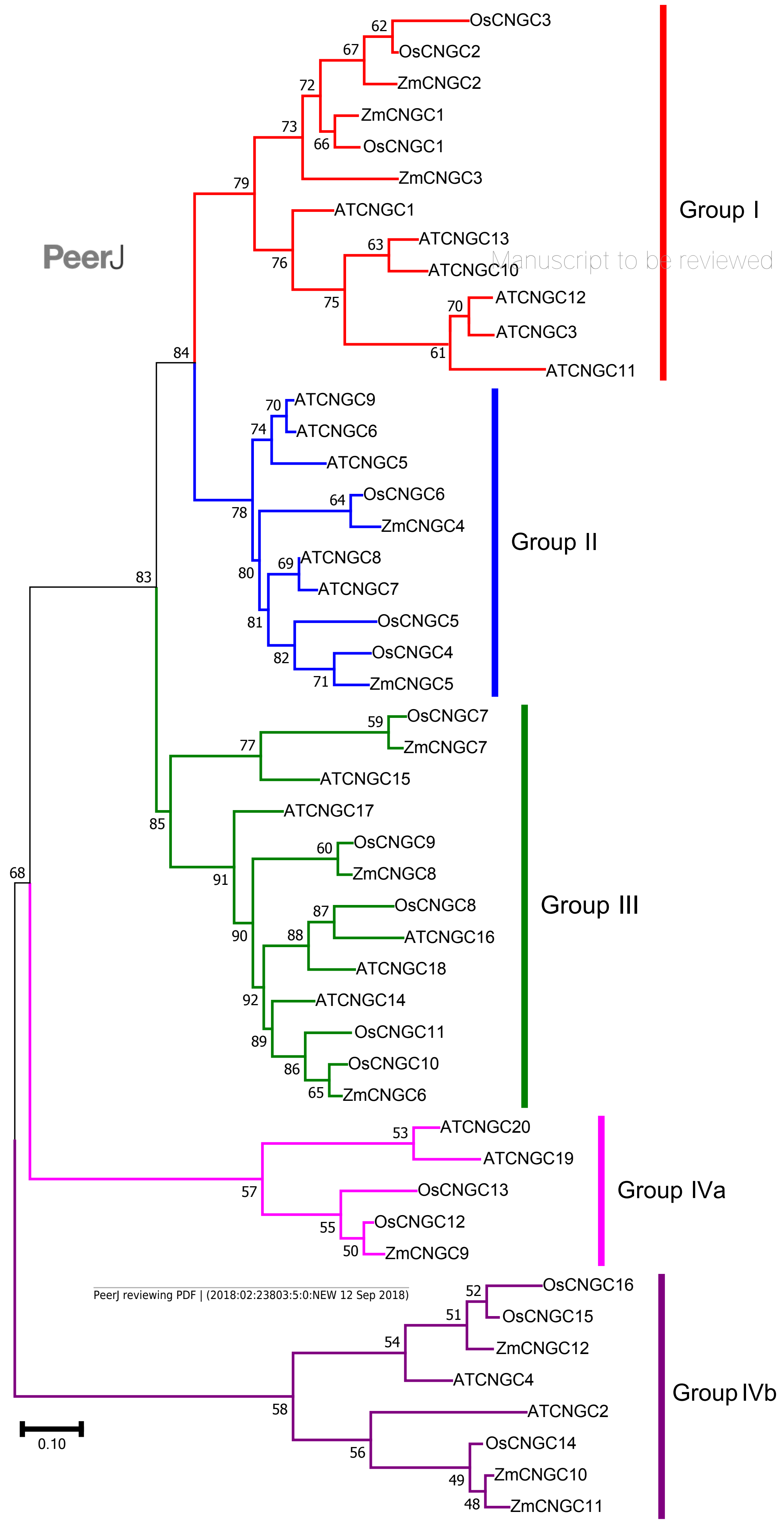




\section{Figure 3}

Phylogenetic relationships (A), motif compositions (B), gene structure (C), and (D) motif logo of ZmCNGCs.

The maximum likelihood (ML) tree under the Jones-Taylor-Thornton (JTT) model was constructed with 1000 bootstrap replications using MEGA7 based on the full-length protein sequence. The exon-intron structures of these genes were graphically displayed by the Gene Structure Display Server using the CDS and genome sequence of ZmCNGC genes. The protein sequences of $\mathrm{ZmCNGC}$ genes were used to predict the conserved motifs by using the MEME Suite web server. 

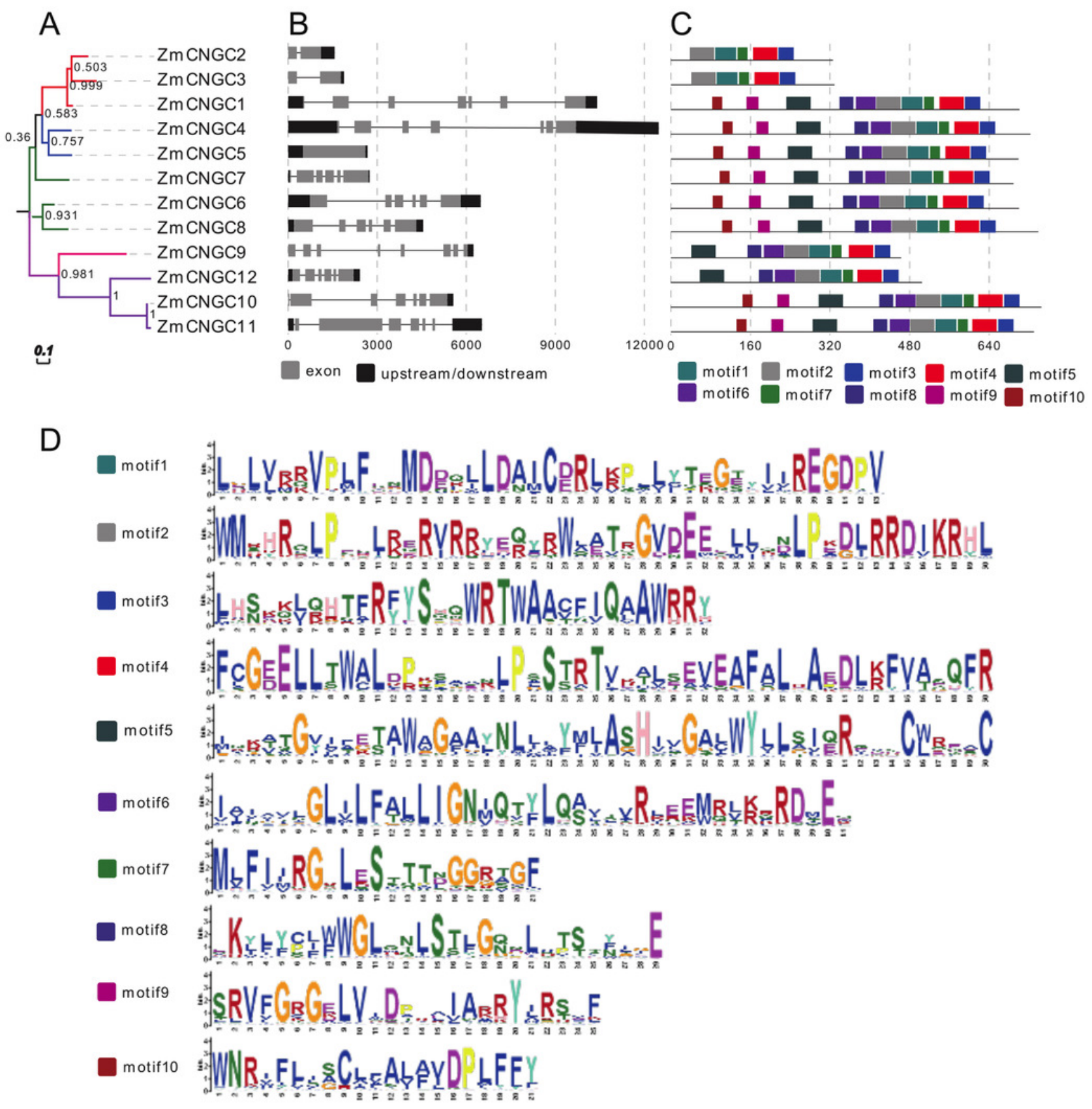
Figure 4(on next page)

The protein-protein interaction network of ZmCNGC genes in maize.

The protein-protein interaction network were constructed based on STRING database. 


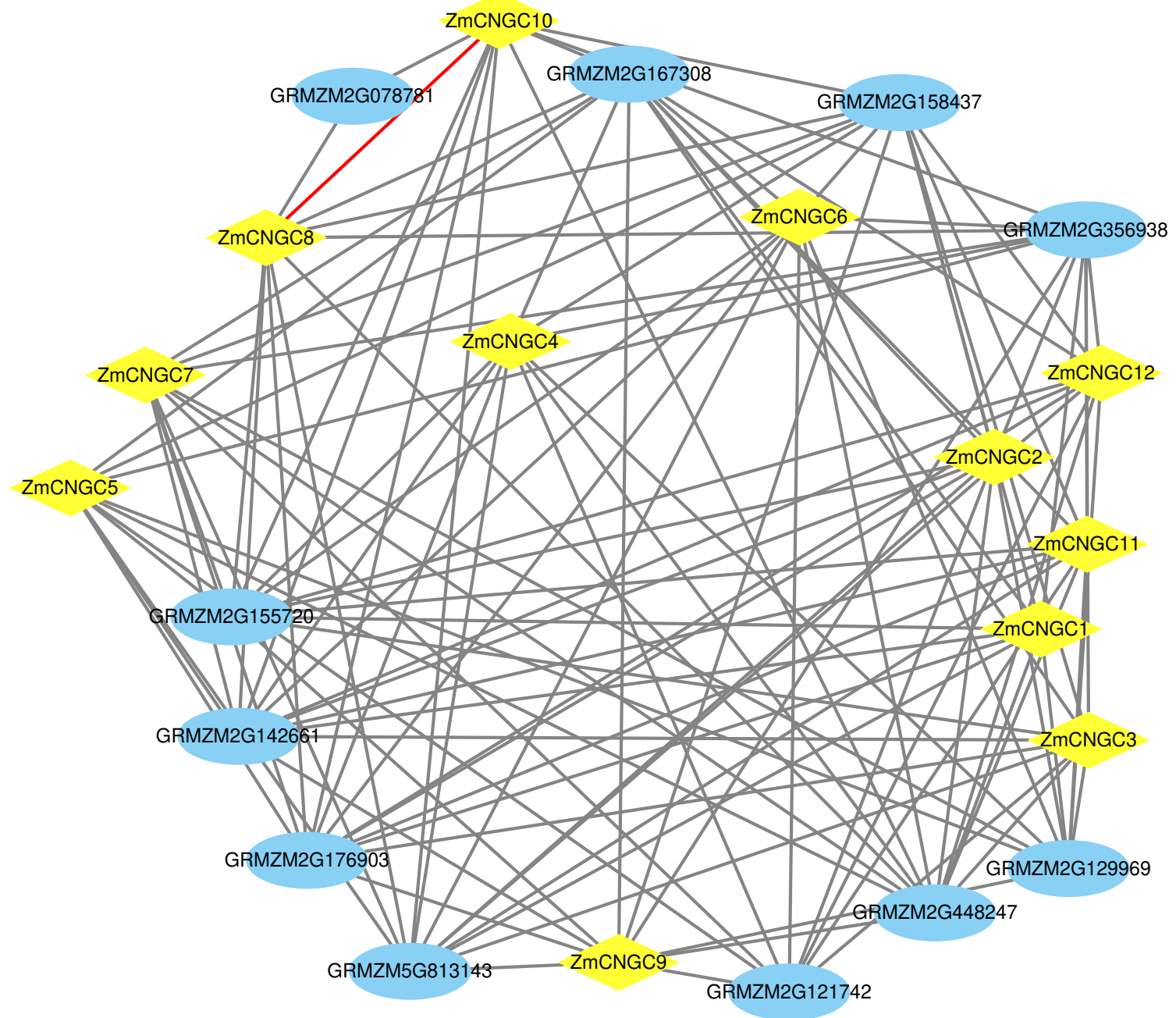


Figure $\mathbf{5}$ (on next page)

Expression profiles of ZmCNGCs in six different tissues and different development stages in embryo, endosperm and seed after pollination, respectively.

(A) Expression profile in six different tissues, including ear, embryo, endosperm, pollen, root and tassel. Expression profile in (B) embryo, (C) endosperm and (D) seed of some days after pollination. 
A

A

B 10 Day

12 Day

14 Day

16 Day

18 Day

20 Day

22 Day

24 Day

26 Day

28 Day

30 Day

32 Day

34 Day

36 Day

38 Day

C 6 Day

8 Day

10 Day

12 Day

14 Day

16 Day

18 Day

20 Day

22 Day

24 Day

26 Day

28 Day

30 Day

32 Day

34 Day

36 Day

38 Day
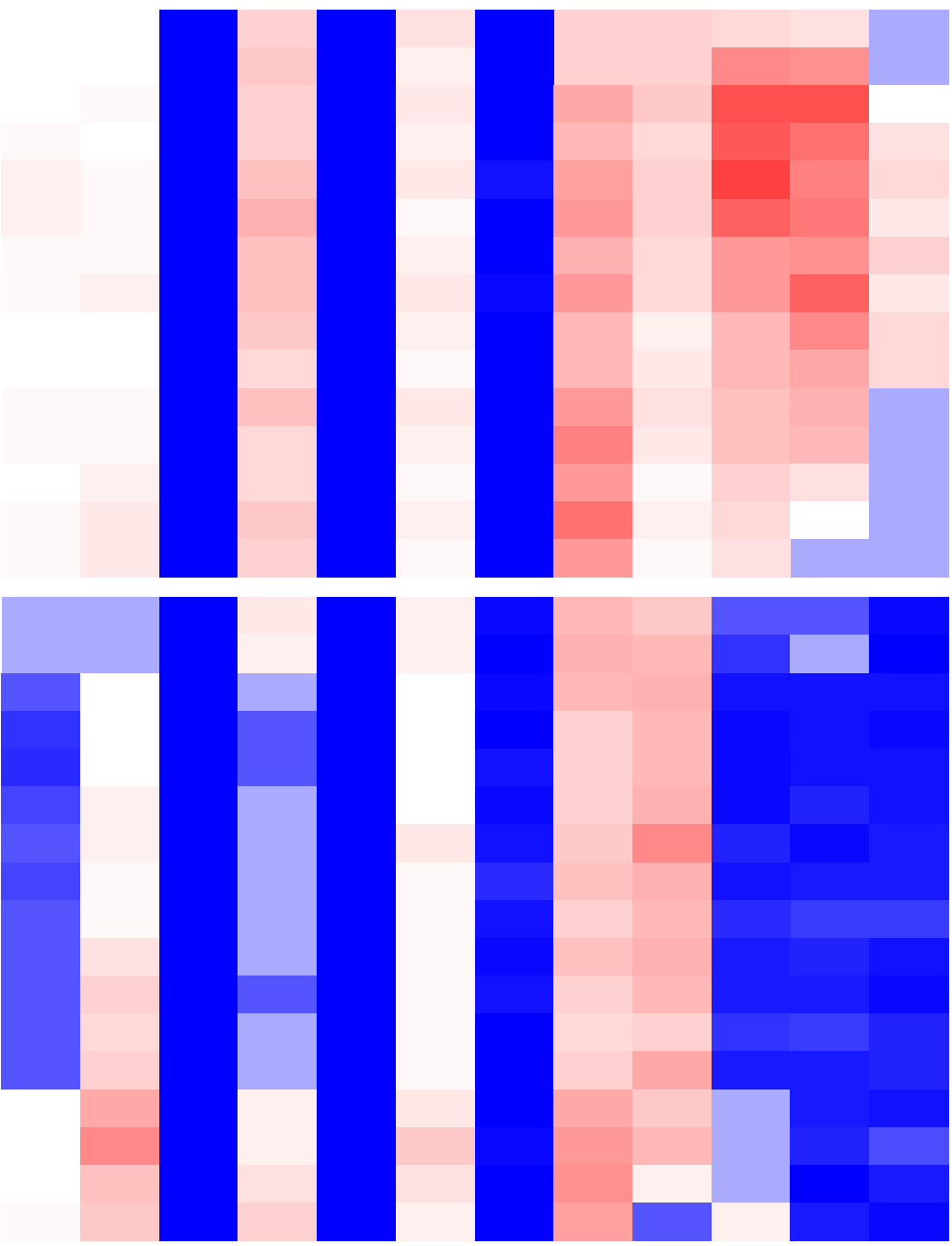

$0 \quad 2$ Day

3 Day

4 Day

6 Day

8 Day

10 Day

12 Day

14 Day

16 Day

18 Day

20 Day

22 Day

24 Day

26 Day

28 Day

30 Day

32 Day

34 Day

36 Day

38 Day
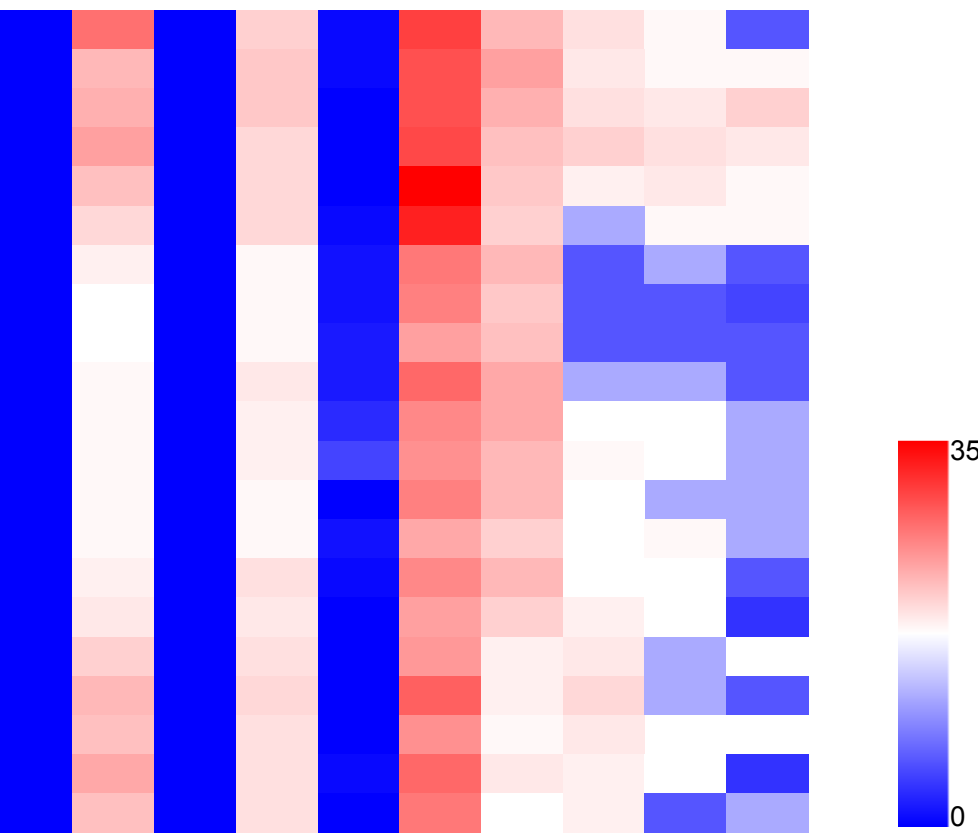